\title{
Research on wireless communication technology based on automatic logistics system of welder
}

\author{
Xuan Sun ${ }^{1, a}{ }^{*}$, Zhi-yong Wang $^{1, b}$ and Zhe-dong $M a^{1, c}$ \\ ${ }^{1}$ School of Mechanical Engineering, University of Jinan, Jinan, China \\ ame_sunx@ujn.edu.cn; b971917165@qq.com; ‘394396041@qq.com
}

\begin{abstract}
In order to meet the requirements of high real-time and high stability of data transmission in automatic welding system, RTU data format and real-time communication mechanism are adopted in this system. In the automatic logistics system through the Ethernet and wireless WIFI technology will palletizer, stacker, AGV car organically together to complete the palletizer automatic crawling the goods, AGV car automatic delivery, stacking machine automatically out of the Dimensional warehouse. .
\end{abstract}

\section{Introduction}

With the continuous development of China's economy, the advantage of lower cost of human resources has gradually disappeared [1]. Higher and higher cost of human resources, more and more Chinese enterprises lost the advantage of market competition, these enterprises urgently need to introduce automated production equipment to replace manpower, reduce costs [2]. The automatic logistics system can not only completely replace manpower, but also greatly increase the efficiency of production [3]. However, for automated logistics systems, wireless communication is the technical difficulty in this automation system. It requires the communication between the system to have higher realtime and higher stability [4]. Therefore, it is urgent to study a wireless communication network environment which can adapt to complex industrial environment, and has higher real-time and stability [5].

\section{Application environment analysis of automatic logistics system for welding machine}

Automated warehouse mainly uses high-level shelves, standard pallets, storage of goods, mainly in three-dimensional warehouse stacker, combined with automated logistics equipment to automate warehouse operations. The supporting equipment mainly includes palletizing equipment, $\mathrm{AGV}$ car and so on. The stacker will be responsible for the

*Xuan Sun: me_sunx@ujn.edu.cn 
production line of packaging machine according to the rules to the stacking goods, AGV to carry the goods put the goods tray to the entrance of the three-dimensional warehouse, warehousing operations by stacking machine. At the same time stacker can manually send out or receive management computer instructions for outbound operations. The overall layout of the system is shown in Figure 1.

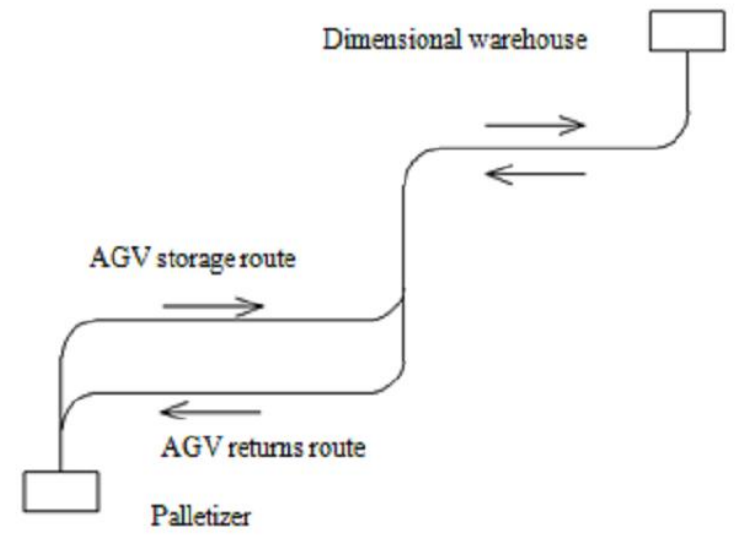

Fig. 1. Layout of automated warehouse system

As the production line of the welder needs to be transported to the distance of more than 300 meters in the three-dimensional warehouse, and in order to reduce the impact of the system on the original production environment, AGV car must run on a specific path. Path in figure 1. Through the AGV car running path, production requirements and cost analysis. Finally, we will use two AGV for welding machine transport operations. As the communication distance is long, and $\mathrm{AGV}$ and stacker are in motion state, the traditional RS232, RS485 and other wired communication cannot meet the system communication requirements, so this system is adopted Ethernet communication, the specific method is: using industrial WiFi signal router to cover the entire plant. Connect the management PC (server), stacker controller, palletizer controller, AGV car and other equipment to the router.

\section{Network structure}

Through the analysis of the on - site environment, the final communication structure of the system is shown in Figure 2.

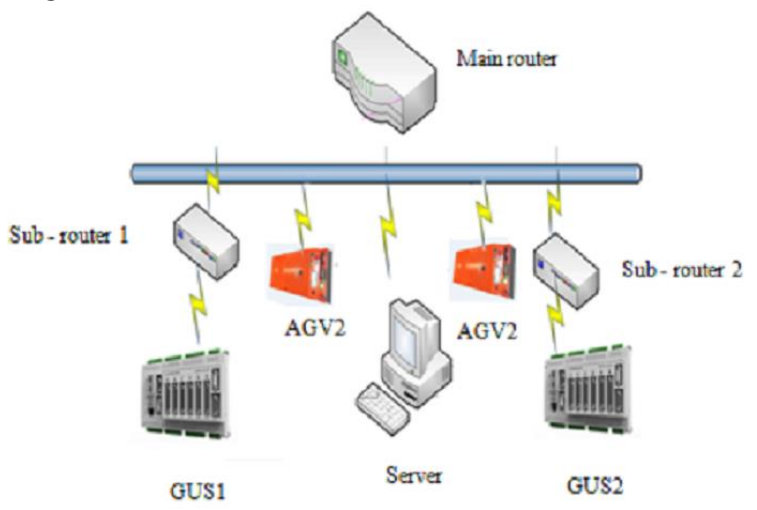

Fig. 2. Communication network diagram of stereoscopic warehouse system 
In Figure 2, with PC as the management system of TCP communication server. Stacking machine, palletizing machine, AGV carrier through their respective Internet access module to access the local area network, real-time management PC to send status, request and other information; management PC to accept the connection of each device, Stacking machine, palletizer, AGV transporter through their respective Internet access module to access the local area network, real-time management PC to send status, request and other information; management PC to accept the connection of various devices, and verify, explain the equipment upload Of the data, so as to obtain the current state of each device. And the corresponding control instructions are sent according to the current system state, so that the other devices can be unified and managed according to the current state of each device. The stacker and palletizer are powered by a motion controller that communicates with the server via an RS232 serial port to the wireless WIFI module. AGV also uses the RS232 serial port to connect to the wireless WIFI module. And the server is directly connected to the Sub - router 2 LAN port, because the server is relatively close to the location of the Sub - router 2[6]. And wired data transmission than wireless WIFI transmission faster and more stable, so that other equipment can make a variety of information more quickly and accurately transmitted to the server.

\section{Application of wireless Wifi technology}

WIFI full name Wireless Fidelit, in essence, is a commercial certification, with Wi-Fi certified products in line with IEEE802.11a / b / g / $\mathrm{n}$ wireless network specifications, it is currently using a more extensive WLAN standard, the usual working frequency is $2.4 \mathrm{GHz}$ [7].

The automatic welding logistics system adopts wireless WIFI technology mainly because of the following aspects:

(1) Construction is convenient and quick. Because WIFI is a wireless technology. So in the construction of a wide range of network eliminates the need for a large number of cabling work, only a certain number of routers, using wireless bridging technology to meet the automatic logistics system requirements of the local area network.

(2) Radio waves transmit farther. At present, the wireless WIFI in the market can be transmitted over 100 to 300 meters without obstacles. However, the transmission distance of wireless Bluetooth is only about 15 meters. But the obstruction of the obstacle greatly reduces the quality of the wireless WIFI signal.

(3) The transmission speed is very fast. At present, routers in the market can reach $37.5 \mathrm{Mbit} / \mathrm{s}$, which can fully meet the needs of the data transmission of the automatic logistics system.

Due to obstruction of industrial equipment, the intensity of the wireless WIFI signal will be greatly reduced and the quality of the data transmission will be affected. Therefore, in a very complex factory environment, a single router has been unable to achieve high efficiency and high quality data transmission. In order to achieve high quality data transmission, we use wireless bridging technology to build a wireless communication environment with large signal intensity and wide coverage. At present, the wireless bridging technology mainly has the following three kinds:

1) Point to point wireless bridging;

Point to point wireless bridging mode can be used to transmit data far from the signal. It is bridged by two wireless routers, thus doubling the distance of data transmission.

2) Point to multipoint wireless bridging; 
The point to multipoint wireless bridging mode enables the decentralized wireless network to be integrated into one entity, and one of the routers acts as the primary router to communicate with all other secondary routers.

3) Bridge relay

Bridge relay is the application of two LAN, there is a certain obstacle to block the transmission of data.

In Automatic Logistics System of Welder, we use a point to multipoint wireless bridging mode, the palletizer, stacker, AGV timely communication with the server, through the analysis of the site environment and AGV trajectory, we use a main router and two sub-router connection mode, the router placed in Figure 3.

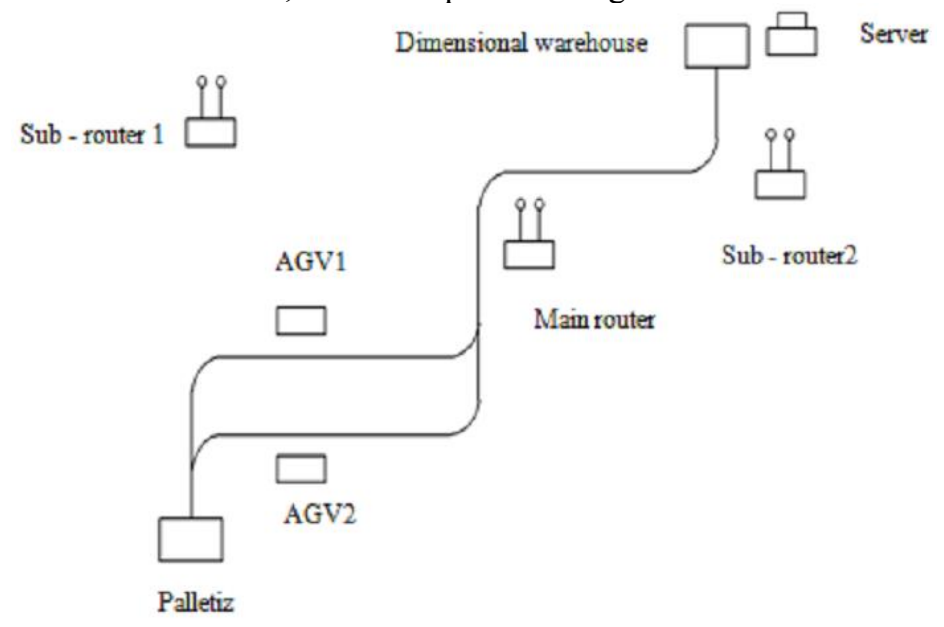

Fig. 3. Location map of router

The three routers shown in the diagram are all installed in the higher position of the factory to reduce the impact of the plant equipment on the signal. In order to enhance the stability of data transmission, all routers have fixed IP addresses, and in order to reduce other foreign equipment to snatch IP addresses, we set up a specific device is allowed to access specific routers. The stacker is closest to the sub router 1, so it is allowed to access, and the AGV trolley will also pass through the coverage of the sub router1, and also allow it to access. So we have to bind the MAC address of the access device to prevent the access of other devices, so as not to affect the stability of the system. The sub router 2 allows only $\mathrm{AGV}$ vehicles to access, but the master router is responsible for bridging the other two sub routers and connecting our servers. Thus, in the process of running, AGV car can choose which router to access according to its own needs, so as to ensure that the AGV car will transmit its status information to the server in time.

\section{Selection of communication protocols}

The network transport protocol mainly includes TCP protocol and UDP protocol, in which the UDP protocol is based on connectionless, which requires lower system resource and high transmission efficiency. However, the UDP protocol sends the information from the Send terminal to the Receiving terminal, but whether the receiver has received the information, it will not be verified, so the disadvantage of the UDP protocol is poor reliability and prone to loss of data packets.

TCP uses connection oriented communication. In the process of communication, it needs to complete the three handshake connection. This three-way handshake is between 
the Receiving terminal and the sender to receive and send data. The three handshake is as follows:

(1)First, establish a connection between Receiving terminal and send terminal. When the sender needs to send data, the sender will send a SYN packet to the receiver, indicating that they will be communication, and then wait for the response of the receiver.

(2)When the receiver receives the SYN packet sent by the sender, it will analyze the packet and send an ACK + SYN packet to the sender according to its own state, indicating that it has received the information and able to communicate.

(3) Finally, when the sender receives the ACK + SYN packet from the receiver, it sends an ACK packet to the receiver. After the packet is sent, the whole connection process is completed. And then the data can be transmitted between the receiver and the sender.

By using the TCP communication protocol, the reliability of data communication is greatly improved. Therefore, communication has been established before sending valid data. For the formal transmission of data laid a reliable foundation. And, TCP has a timeout retransmission mechanism and data validation mechanism, can guarantee the reliability of data transmission.

In the welder logistics system, because less transmission data, but the data reliability requirements are higher, so the choice of TCP transmission protocol. Through the analysis of the communication information between the equipment of the automatic logistics system of the welder, the response protocol is adopted, and the RTU data format is adopted on the basis of ensuring the convenience of programming. The data structure is shown in Table1.

Table 1. Communication protocol

\begin{tabular}{|c|c|c|c|c|c|c|c|}
\hline Byte & 1 & 2 & 3 & 4 & 5 & 6 & 7 \\
\hline Data name & Start & Start & $\begin{array}{c}\text { Source } \\
\text { device }\end{array}$ & $\begin{array}{c}\text { Data } \\
\text { length }\end{array}$ & $\begin{array}{c}\text { Control } \\
\text { command }\end{array}$ & $\begin{array}{c}\text { Control } \\
\text { parameter }\end{array}$ & check \\
\hline Data & 0x5A & 0xA5 & & $0 \times 03$ & & $\begin{array}{c}5 \text { and } 6 \text { bytes } \\
\text { XOR }\end{array}$ \\
\hline
\end{tabular}

The data is parsed as follows:

Start: 0x5A, 0xA5, Because WIFI wireless communication, the first two bytes of data often error, so the first two bytes of data transmission are $0 \times 5 \mathrm{~A}, 0 \mathrm{xA} 5$, used to avoid data transmission errors.

Source Device Number: Used to indicate the device that is transmitting information. 0x01 indicates the palletizer controller (GUS1), 0x02 represents the three-dimensional warehouse controller (GUS2), 0x03 represents the three-dimensional warehouse controller (turntable 3), 0x04 stands for AGV1, 0x05 means AGV2, 0x06 indicates the incoming PC (server).

Data length: there are 3 bytes after $0 \mathrm{x} 03$ bytes, for the server to verify the data.

Control command: 0x01 indicates that the request to enter the turntable instructions, 0x02 indicates AGV reach the specified location of the instructions.

Control parameters: When the control instruction is $0 \mathrm{x} 01$, the control parameter is $0 \mathrm{x} 01$, said the request to enter the No. 1 turntable instructions.

Check: XOR of control command and control parameter.

Because the control instruction segment and the control parameter segment are the parameters and commands that the server and the lower computer need to execute, it is necessary to ensure the correctness of the data, and the other data has little effect on the execution of the program.

When the sender sends data, the control instruction and the control parameter are XORed, and then the operation result is put into the check byte. When the receiving end 
receives data, the control instruction and the control parameter are XORed and the operation result is compared with the accepted check byte. If the data is the same, the receiver will then parse the data and send the response data to the sender. If the data is different, the receiver will not parse the data and will not send the response data. After a certain period of time, if the receiver cannot receive the response data will be re-sent to the receiver the same data. If the sender retransmits 5 times the data still does not receive the response data will be alarm, but the sender will continue to retransmit the data. If you reissued 1000 times, still cannot receive the answer to stop sending, waiting for processing. After receiving the response will automatically terminate the alarm and perform the next action. Through this data verification to ensure the accuracy of the data, making the whole system more secure and reliable.

When the server receives the following data: 0x5A, 0xA5, 0x04, 0x03, 0x01, 0x02, 0x03, Indicates that AGV1 requests access to turntable 2.

Using this data format, the sender and the receiver duplicate check data ensure the correctness of the data. 7 bytes of data length not only to complete the welding automatic logistics system of various information transmission needs, and as much as possible to shorten the length of the data to improve the efficiency of data transmission, and meet the system requirements of high real-time.

\section{Message transfer mechanism}

According to the welding machine production rate analysis, the production process must be two AGV work at the same time to meet the production requirements. In order to prevent two AGV collision, AGV operation must be unified planning by the server. This requires the server to be able to understand the specific location of each AGV. After analyzing the running path of $\mathrm{AGV}$, six important positions are finally determined, as shown in Figure 4. In these six positions, the IC card is fixed as the logo of the six positions. When the AGV arrives at each card, the location information is sent to the server. The server obtains AGV location information to accurately control the operation of $\mathrm{AGV}$, palletizer, stacker, thus making the whole system safe and efficient operation.

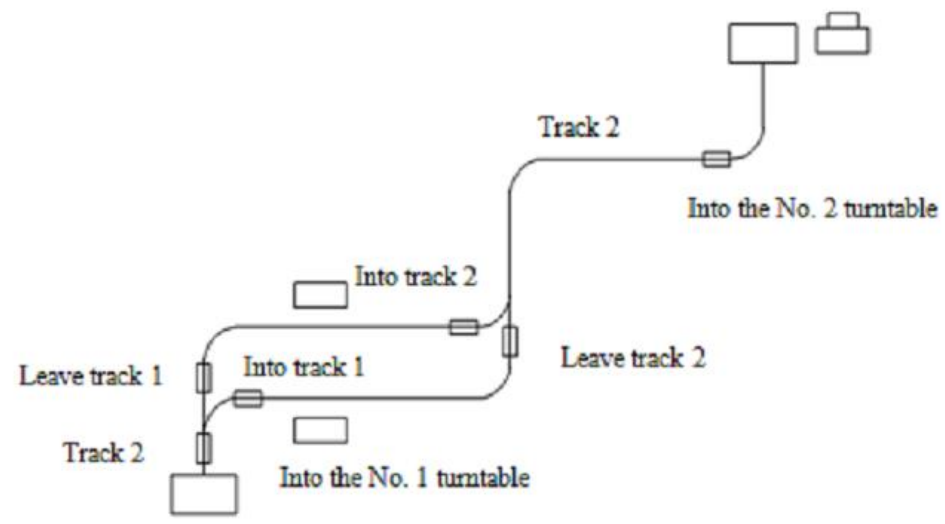

Fig. 4. IC card location diagram

With the AGV movement, the connection will be transformed between several routers. Making the connection time longer. Location information cannot be sent to the server in a timely manner. And in the complex environment, some wireless signal interference will temporarily interrupt communication of $\mathrm{AGV}$, palletizer, stacker with server. So when $\mathrm{AGV}$ and server communication is interrupted, $\mathrm{AGV}$ will stop and wait, and will continue 
to send information to the server, the AGV was not restarted until the server received the information. If the server has not answered successfully after sending a certain number of messages, the system will alarm, so that the whole system is more secure operation.

Among them, two AGV through the wireless serial port conversion device to achieve serial signal conversion to wireless Ethernet signals, palletizer and three-dimensional warehouse through the wireless WIFI module will be wired Ethernet signal into wireless Ethernet signal. The management computer obtains the wireless Ethernet signal through the wireless network card. The device is simultaneously connected to the same network node. And then through the response mechanism to establish a connection. In order to achieve the system scheduling and monitoring.

In the communication network, there are five devices, namely, management computer, AGV1, AGV2, three-dimensional warehouse stacker, palletizer, the basic communication requirements are as follows: Among them, in order to achieve storage control, AGV1, AGV2, three-dimensional warehouse Stacking machines, palletizing machines communicate with the server. PC communicate with the warehouse stacker, and to complete the operation of the product into the warehouse.

In order to achieve communication requirements, there are two basic programs, one is not unified management between the equipment. Each device only establishes a connection when it is needed to send a message. Communication is complete, and close the connection. When AGV1 reaches the station of request to enter the turntable 2 will send a request to the server. Then the server issues a query command to the stacker controller. The stacker sends instructions to the server according to its own status. When the server receives the stacker information and then decide whether to allow AGV1 into the No. 2 turntable and send information. AGV1 performs the action after receiving the server's information. The advantage of this transmission mechanism is a small amount of data and the devices are independent of each other. Because data is sent when data is needed, and resulting in realtime poor, inconvenient unified monitoring of the state. Second, through the wireless WIFI built LAN, each device continually sends status information to the server, called a real-time communication mechanism. When the AGV sends a variety of request information, the server sends instructions directly to the AGV based on the received information. When AGV1 issues 0x5A 0xA5 0x04 0x03 0x01 0x02 0x03 (AGV1 requests to enter the No. 2 turntable) information, the server replies directly according to the received information.

When the AGV1 arrives at the request of the No. 2 turntable station, the server sends a direct command to allow AGV1 to enter the No. 2 turntable based on the palletizer status information that has been received. At the same time in order to be able to real-time monitoring of each device and server connection, the device will send an online information to the server every 5 seconds, indicating good communication with the server. When the server cannot receive the equipment online information will alarm, improve the reliability of the entire system. The advantage of this communication mechanism is a small amount of data. convenient unified management, monitoring, and good real-time.

In order to improve the system's real-time and easy management, the communication system using the second program. That is, the management of the computer as a LAN server, the rest of the equipment as a client, and the management of the computer to establish a connection. In order to reduce the number of inquiries, improve communication efficiency. Each device immediately uploads status to the server after the status changes. To facilitate the server to accurately determine the system status, issued the correct order.

\section{Conclusion}

Welding automatic logistics system using wireless WIFI technology to build a local area network, and to complete the data transmission between the various devices. Through the 
field test, the system, that uses RTU data format and real-time communication mechanism, fully able to meet the high real-time and high stability of the data transmission requirements.

\section{References}

[1] Xiaohui Wei, Meizhen Yang. Wireless WiFi technology application analysis. Modern commerce industry. 2013 (18): 26-32. In Chinese.

[2] Yang Li. WiFi technology principle and application research. Science and Technology Information. 2010 (06):56-62.

[3] Chunfei Zhang. WiFi technology principles and future trends. Digital Community \& Smart Home. 2008 (11):182-186.

[4] Tao Wang, Introduction to Wireless Network Technology. Qinghua University Press, 2008.

[5] Qingjiang Jin,Wireless network technology and application. Shanghai Jiaotong University Press, 2003.

[6] Kyung-Chang Lee,Hyun-Hee Kim.Communication Delay Properties in Performance Model of Profibus Token Passing Protocol. Proceding of the 7th Korea-Russia International Symposium 2003.24:234-242.

[7] Y. F Wang, C. H Wang, X Huang. Guaranteed cost control with random communication delays via jump linear system approach. The 8th International Conferenceon Control, Automation, Robotics and Vision. 2004.23:180-186. 